\title{
Una mirada hacia el proceso de identidad en el valle de Toluca precortesiano, México
}

por

\author{
Yoko Sugiura Yamamoto, César Villalobos Acosta, M. ${ }^{a}$ Del Carmen Pérez \\ y Elizabeth Zepeda \\ UNAM/IIA
}

\begin{abstract}
En este artículo se explora la identidad como un proceso cultural de larga duración en el que intervienen factores culturales en diferentes niveles. Se argumenta que tanto la hegemonía como el declive de Teotihuacan influyeron en el desarrollo de las identidades colectivas en el valle de Toluca. El estudio de la identidad es de particular importancia. Se considera pertinente que, a través de la cultura material, especialmente de la cerámica arqueológica, se profundice en el complejo entramado hacia la consolidación de la identidad.
\end{abstract}

Palabras clave: identidad; Teotihuacan; valle de Toluca; Epiclásico y Posclásico.

\section{INTRODUCCIÓN}

La identidad constituye un tema fundamental que se ha tratado de manera constante en diversas disciplinas científicas ${ }^{1}$. Su importancia se reconoce por el hecho de que los individuos y grupos sociales negocian constantemente sus relaciones con el fin de marcar diferencias y situarse dentro de un «nosotros» o «ellos» ${ }^{2}$. Si bien ésta representa una gran diversidad de significaciones, la más sencilla y simple acepción se refiere a las formas en las que los individuos y los grupos se distinguen, a su vez, en sus relaciones sociales con

\footnotetext{
${ }^{1}$ Bartra, 1987. Hooson, 1994. Spencer, 1994. Cerulo, 1997. Du Gay, et al., 2000. Huddy, 2001. Schortman, et al., 2001. Sökefeld, 2001. Chorba, 2007.

2 Jenkins, 1996. Meskell, 2001; 2002. Díaz-Andreu, et al., 2005.
} 
los otros ${ }^{3}$. Dado que por sus características inherentes es multi-dimensional y multi-vocal, la identidad se conjuga en torno a prácticas sociales que están siempre dentro de un proceso dinámico y relacional ${ }^{4}$.

La identidad se gesta y desarrolla en circunstancias históricas e institucionales concretas y se modifica permanentemente. Es por ello que resulta difícil adscribirse a una definición estática de la misma, y que puede ser entendida, de diversas maneras, como la filiación individual o colectiva, bajo reconocimiento explícito o implícito, de diferencias socialmente aprobadas como significativas $^{5}$. Así, la identidad entendida como un fenómeno (o proceso) dinámico no es estático ni de naturaleza innata, sino fruto de negociaciones sociales concretas $\mathrm{y}$, por ende, adaptable a condiciones cambiantes, ya sean personales o colectivas.

Es por ello, que en escenarios particulares, ya sean de larga o corta duración, los grupos e individuos, tengan la necesidad adscribirse, cuestionar o externar su identidad con respecto a «otros». Como resultado se tiene que la auto-adscripción se vincula con acontecimientos históricos que justifican ya sea estatus, genealogía, etnicidad o nacionalidad, por sólo citar algunos. El proceso de identidad es una construcción multi-causal en torno a un conjunto de prácticas iterativas siempre contingentes, dinámicas y heterogéneas. Además, dicho proceso dinámico surge $\mathrm{y}$, al mismo tiempo, es resultado, de la conjunción de estrategias colectivas e individuales. Es pertinente señalar que el sentido de la identidad se exhibe, por regla general, con mayor intensidad en momentos de tensión social o política, como en el caso del presente estudio que discutimos más abajo ${ }^{6}$.

\section{LA NOCIÓN DE IDENTIDAD EN ARQUEOLOGÍA}

El interés por el estudio de la identidad en arqueología tiene una larga historia, en la cual se distinguen tres posturas teóricas como las principales. La primera de ellas, desarrollada como parte de la corriente histórico-cultural, predominó en la primera parte del siglo XX, en la cual se enfoca el concepto desde una perspectiva esencialista, es decir, la identidad como una cualidad adscrita con la que un ser humano nace. La segunda, conocida como arqueología procesual, en boga desde de la década de 1960, caracteriza la cultura

\footnotetext{
3 Jenkins, 1996: 4.

${ }^{4}$ Insoll, 2007: 13.

5 Jenkins, 1996. Meskell, 2001; 2002. Díaz-Andreu, et al., 2005.

${ }^{6}$ Graham, 1994. Foote, et al., 2000. Clark, 2005. Kaya, 2005.
} 
como un sistema, en donde el estudio de las identidades queda relegado a un segundo plano. Finalmente, la tercera, consolidada en la década de 1990, denominada como arqueología postprocesual o interpretativa, conceptualiza a la identidad como un proceso integral, para cuya comprensión es necesario no sólo tomar en consideración los contextos particulares, sino también ponderar el papel que juega el sujeto en dicho proceso.

La perspectiva esencialista entiende la identidad como una cualidad innata al ser humano. Se reproduce a través de normas sociales y se caracteriza por una continuidad histórica-evolutiva. Esta idea implícitamente sostiene que los individuos actúan de acuerdo con preceptos regulados y justificó que algunos atributos como lengua y características físicas o rasgos culturales y geográficos, se hayan utilizado para definir la identidad y, de ahí, equipararla con el concepto de etnicidad ${ }^{7}$. En arqueología, esta postura teórica contribuyó en la construcción de uno de los paradigmas más importantes conocido como histórico cultural ${ }^{8}$, cuya aportación en términos del acopio de datos sentó las bases para la identificación de áreas culturales y sus rasgos característicos. De acuerdo con esta postura, la correspondencia entre la cultura material y sus hacedores denota, de alguna manera, grupos étnicos o identidades específicas ${ }^{9}$.

A partir del neopositivismo hempeliano, utilizado en arqueología desde finales de la década de 1960, se plantearon cambios en la forma de abordar la cultura material, y por tanto la concepción de identidad. Una de las contribuciones más sobresalientes de dicha corriente se atribuye al desarrollo de una metodología científica mediante la cual los arqueólogos manejan los datos bajo estudio más allá de las clasificaciones descriptivas ${ }^{10}$. Además, en el esquema procesual, se considera que la sociedad se constituye fundamentalmente como un sistema general y es analizable objetivamente a partir de una mirada cartesiana $^{11}$, el papel del individuo como generador de cambios sociales, y con ello el proceso de identidad, es minimizado y visto de manera pasiva.

Por su parte, la consolidación de los postulados de la arqueología postprocesual en la década de 1990 fomentó la idea de que la cultura no es extra somática a los seres humanos, sino por el contrario, se gesta como parte de un proceso relacional, dinámico y contextual, que incluye aspectos cognitivos. A la par del desarrollo de esta corriente, los estudios en torno a la identidad se

\footnotetext{
7 Sugiura, 1991.

8 Jones, 1996: 62-80.

${ }^{9}$ Lyman, et al., 1997.

10 Binford, 1983. Binford y Binford, 1968. Hammond, 1971. Watson, et al., 1971. Schiffer, 1976. Sabloff, 1998.

11 Watson, et al.,1971. Gándara, 1981.
} 
colocaron como uno de los intereses principales en las ciencias sociales ${ }^{12}$, en los cuales se reconoce la gran complejidad y diversidad sociocultural anteriormente ignorada ${ }^{13}$. Dentro de esta postura, algunos ponderan, por ejemplo, al papel económico en la configuración de las fronteras, mientras que otros enfatizan la importancia en algún interés particular con respecto a los recursos políticos y económicos. Otros señalan las necesidades de reconocer el papel de individuo, así como la identidad del grupo como fluida, circunstancial y no adscrita ${ }^{14}$.

En resumidas cuentas, la arqueología interpretativa analiza la identidad en su naturaleza multidimensional ${ }^{15}$, el cual se forja mediante una negociación relacional entre «nosotros» y «ellos». Así, la identidad étnica o pertenencia a un grupo social se construye de manera histórica, dinámica y circunstancial ${ }^{16}$. En otras palabras, la formación y transformación de la identidad es contingente en relación con las condiciones históricas particulares ${ }^{17}$. Resulta claro por tanto, que ésta no se puede comprender simplemente enlistando rasgos particulares ya sea culturales o lingüísticos ${ }^{18}$. Se podría afirmar, entonces, que la consolidación de los postulados de la arqueología interpretativa fortaleció la idea de que la cultura es parte de un proceso dinámico y contextual, en el que las identidades individuales y colectivas son constitutivas de complejas iteraciones sociales ${ }^{19}$.

En el presente artículo, el interés se centra en la perspectiva de observar a la identidad en su dinámica temporal y contextual. Se particulariza en torno a la identidad en tiempos precortesianos a través de la cultura material, y la forma en que ésta podría reflejar el complejo proceso de construcción y trasformación de las identidades. Asimismo, se analizará dicho proceso complejo e intricado a partir principalmente de dos aspectos: la identidad regional del valle de Toluca, inserta en una escala mayor, en este caso concreto del valle de México, con la cual ha establecido una estrecha relación a lo largo de tres milenios de su historia (figura 1) y la identidad colectiva como autodefinición,

12 Smith, 1990; 1991; 1994a; 1994b. Anderson, 1991. Anderson y Andrew, 1994. Hutchinson y Smith, 1994. Cerulo, 1997.

13 Dresser, 1989. Llobera, 1989. Samuel, 1989. Fleury-Ilett, 1993. Escolar, et al., 1994. Hooson, 1994.

14 Sugiura, 1991.

15 Kaya, 2005.

16 Doremus, 2001. Fowler, 2001. Gosden, 2001. Meskell, 2001. Sökefeld, 2001. Holtorf, 2002. Kane, 2003. Clark, 2005. Díaz-Andreu, et al., 2005.

17 Jones, 1996: 75.

18 Jones y Graves-Brown, 1996: 6.

19 Hodder, 1982a; 1982b; 1986; 1987; 1991a; 1991b, 1998; 1999. Shanks y Tilley, 1987a; 1987b. Tilley, 1989; 1999. Shanks, 1992. Hodder, et al., 1995. 
Figura 1. Mapa de localización del valle de Toluca

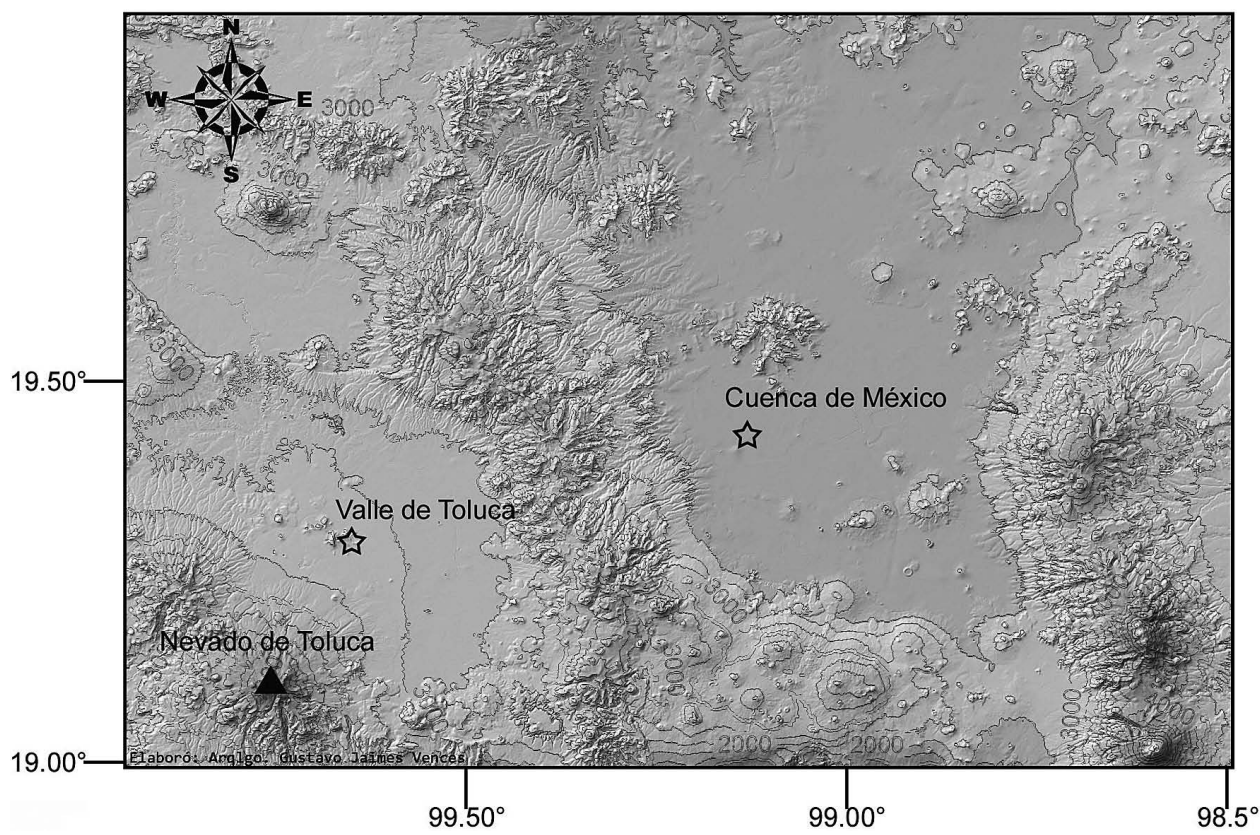

Fuente: elaboración propia.

propia de la región a la cual pertenece. Naturalmente dicha noción comprende un campo de investigación muy amplia. En este trabajo, se abordará a partir de la cultura material de distintas características con énfasis en la cerámica arqueológica, recuperadas en el valle de Toluca, la cual pudo haber repercutido en los procesos hacia la gestación de identidad ${ }^{20}$.

Primeros asentamientos en el Valle de Toluca y SU vínculo histórico CON LA CUENCA DE MÉXICO

Si bien el vínculo social, político y cultural entre la cuenca de México y el valle de Toluca se fortaleció a raíz del surgimiento de Teotihuacan, éste se remonta a tiempos mucho más anteriores, atribuido principalmente a la

${ }^{20}$ Sugiura, et al., 1997. Sugiura, 1998a; 1998b; 1998c; 2001; 2005a; 2006; 2009a; 2009b; en prensa b. Sugiura et al., 2006. Sugiura, Barba, et al., 2010. Sugiura, Zepeda, et al., 2010. Encastin, 2012. Parsons y Sugiura, 2012. 
cercanía física y a la población biológicamente emparentada. Prueba de ello es que, a partir de las primeras aldeas dispersas en el valle de Toluca hace más de 3000 años, ya se manifestaba dicha relación en la cultura material. Aún en la actualidad sigue mostrándose en múltiples aspectos de la vida de los habitantes. De esta manera, la relación de ambas regiones ha perdurado a lo largo de su milenaria historia.

El estudio del patrón de asentamiento señala que, ya en el período Formativo temprano aproximadamente hacia el $1.200 \mathrm{aC}$, el valle de Toluca estaba habitado por grupos sedentarios ${ }^{21}$ (figura 2), en aldeas pequeñas y dispersas en la parte baja de las planicies y terrenos cercanos al rio Lerma y sus afluentes. Al parecer estas aldeas mantenían relaciones de reciprocidad y equidad, sin grandes muestras de diferenciación. Al paso del tiempo, durante el Formativo medio (1.000-500 aC), se incrementa paulatinamente el número de sitios, los cuales se distribuían en la parte central del valle, en la que actualmente se encuentra la capital del estado, pero alejados de las zonas lacustres y boscosas. Para entonces, la agricultura ya se había establecido plenamente, pero sus técnicas incipientes no representan cambios fundamentales. Prueba de ello es el hecho de que siguen utilizándose las zonas de pendiente suave, aunque se registra colonización de nuevas áreas como consecuencia del incremento en el número de asentamientos de baja jerarquía. Asimismo, la cultura material, específicamente hablando de su composición cerámica, incluyendo figurillas, sugiere que los primeros aldeanos del valle llegaron desde la vecina cuenca de México y que, desde entonces, la región toluqueña se insertó en la esfera cerámica del Altiplano Central en la cual la cuenca de México tuvo un papel importante. Esta cercanía podría fortalecerse aún más si consideramos que ambas regiones compartían un origen bio-étnico otomiano ${ }^{22}$.

En el Formativo tardío (400 aC-150-200 dC), sobre todo hacia los finales del mismo, el valle de Toluca registró algunos episodios de virtual abandono, durante el cual disminuyó drásticamente el número de sitios, así como su tamaño. El mismo fenómeno se manifiesta, también, por un empobrecimiento notable en manifestaciones culturales. Se conjetura que el gestante estado Teotihuacan fue una de las causas principales del gran desplazamiento poblacional que dejó virtualmente abandonado el valle de Toluca. La región, como consecuencia, permaneció en condiciones de marginalidad con fuertes tendencias rurales, lo cual contrasta con lo sucedido en la vecina cuenca de México, donde la aparición de centros rectores importantes marcó un proceso definitivo hacia una mayor complejidad social.

${ }^{21}$ Sugiura, 1998. González de la Vara, 1999; 2011.

22 Buentello, et al., 2009. 
Figura 2. Cronología en la Cuenca de México, Valle de Toluca Y TEOTENANGO

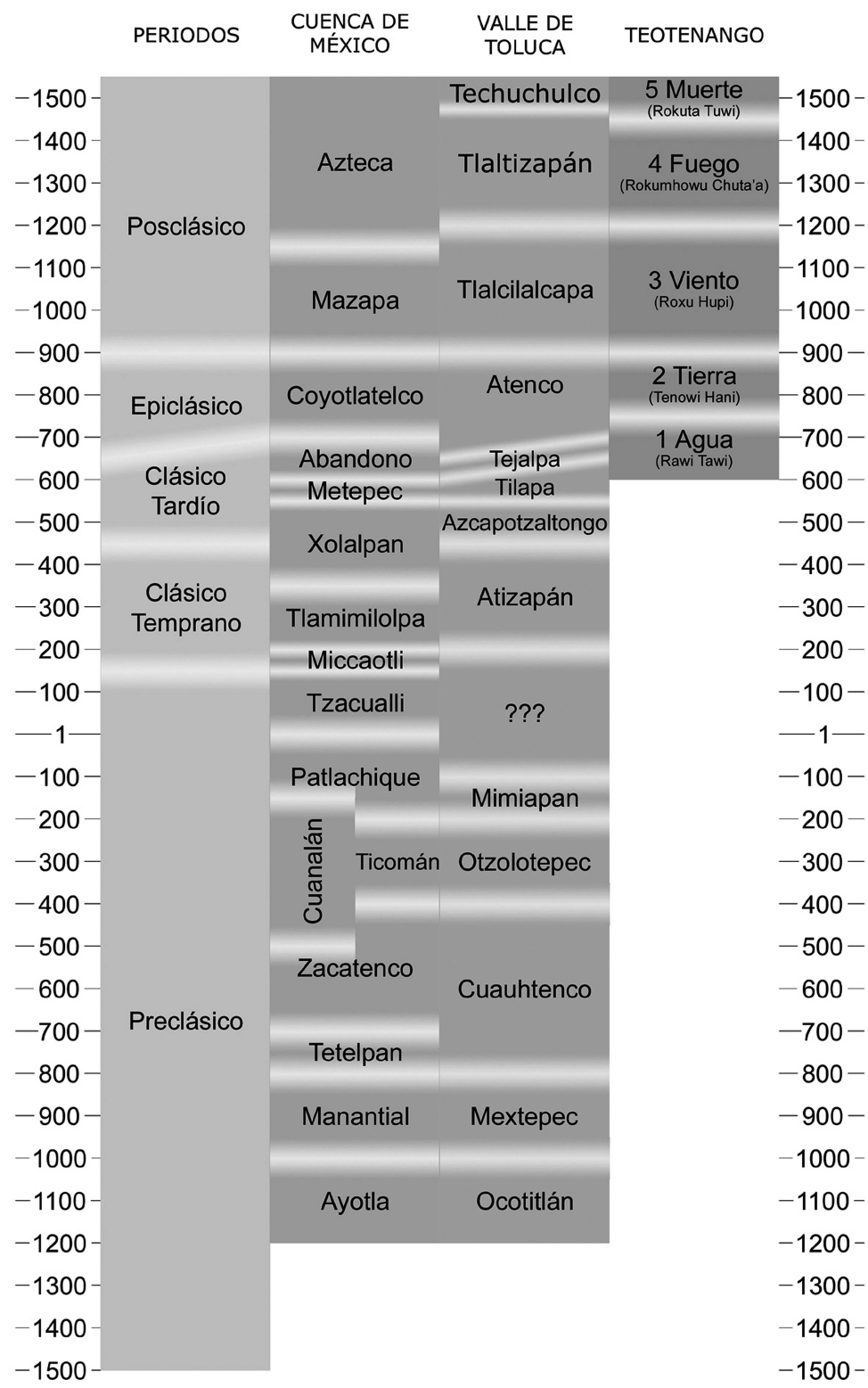

Cuenca de México- Rattray, 1991; 2001. Beramendi-Orosco et al, 2009. Sanders, 1986; 1989. Valle de Toluca- Sugiura, 2005; 2006.

Teotenango- Piña Chán, 1975. 
El valle de Toluca frente al mundo teotihuacano

Después de un corto hiato histórico, el valle de Toluca logró recuperarse de su marginalidad fortaleciendo nuevamente los vínculos con la vecina cuenca de México. Para el Clásico medio, Teotihuacan se había convertido en un gran estado, cuyo poder político y económico se manifestó en la mayor parte del actual territorio mexicano. Conforme avanzaba la consolidación y la expansión política de Teotihuacan ${ }^{23}$, su estrategia política apuntaba a la incorporación del fértil valle de Toluca bajo su esfera político-económica. En este contexto, se aceleró el proceso de repoblación del valle de Toluca. Así, conforme Teotihuacan creció como estado preeminente y mantuvo, durante cientos de años, su poder pan-mesoamericano, su dominio dejó huellas indiscutibles en las zonas, sobre todo, circunvecinas al valle de México, como la región de Toluca. La clara tendencia de la llegada de las poblaciones que provenían de la cuenca de México, probablemente del valle de Teotihuacan, con su legado cultural de aquella metrópoli puede interpretarse como resultado de la adhesión de la región de Toluca al macro-sistema teotihuacano durante la fase Atizapán (ca. 200-400 dC).

Durante los siguientes siglos, el dominio teotihuacano siguió repercutiendo en el desarrollo social, político y cultural en las regiones aledañas. Así, a partir del tiempo correspondiente a las fases de Tlamimilolpa y Xolalpan temprano de Teotihuacan, se vuelve cada vez mayor el vínculo con la urbe y el dominio teotihuacano siguió repercutiendo en el desarrollo social y político de las regiones aledañas. Éste refleja en el acelerado ritmo de crecimiento poblacional en la región del valle de Toluca ${ }^{24}$, el cual no puede explicarse, por completo, por el factor endógeno, sino que es necesario tomar en consideración el exógeno, es decir, la población proveniente de la vecina cuenca de México.

La relación entre el poder político, y la región incorporada a éste como el caso que particularizó Teotihuacan con el valle de Toluca, se manifiestan en múltiples dimensiones en las cuales la cultura material constituye una de las bases fundamentales para dilucidarla. En efecto, como se mencionará a lo largo del presente estudio, la cultura material pareciera apuntar que el legado cultural teotihuacano no sólo fue adoptado, sino también asimilado por las sociedades toluqueñas, fenómeno que puede interpretarse como resultado de la inserción de la región al macro-sistema regido por la llamada Ciudad de los Dioses, así como por el sentir de «cercanía» a los habitantes de dicha metrópoli 2008 .

${ }^{23}$ Gamio, 1922. Millon, 1964. Manzanilla, 1999. Rattray, 2001. Cowgill, 2008. Smyth,

${ }^{24}$ González de la Vara, 1998. Sugiura, 1998. 
por su filiación bio-étnico compartida, vínculo que nunca dejó de existir a lo largo de la historia tanto del valle de Toluca como la de la cuenca de México.

La expansión del poder supra-regional de Teotihuacan durante el horizonte Clásico, sobre todo a partir de hace alrededor de $300 \mathrm{dC}$., se manifiesta, junto con el crecimiento poblacional del valle de Toluca, en una mayor penetración de pautas culturales de dicha urbe. Así, fueron testigos de dicho fenómeno una serie de elementos: el estilo y patrón arquitectónicos, incluso de sus técnicas constructivas y de orientación, que siguen fielmente los cánones teotihuacanos ${ }^{25}$; manifestaciones estéticas en la cerámica ${ }^{26}$ y escultura de piedra; mecanismo de obtención y técnicas de elaboración de artefactos líticos, sobre todo obsidiana ${ }^{27}$, así como uso simbólico de determinados materiales como pizarra y mica. La influencia y/o injerencia teotihuacana se detecta no sólo en los materiales que pertenecen al ámbito político y económico, sino también en los inventarios más elementales de menaje cotidiano. Así, los testimonios de su gran poder se encuentra hasta en una simple olla o cazuela, así como en los cajetes de barro que sirvieron para inventarios más elementales de menaje cotidiano, naturalmente utilizando las materias primas locales que se encontraban a su alcance. Así mismo fueron transmitidas las normas o códigos estéticos y simbólicos en la elaboración de materiales relativos al mundo ritual, a la cosmovisión e ideología, como son los casos de adornos de braseros e incensarios y figurillas de barro ${ }^{28}$.

Así, el escenario que caracterizó el valle de Toluca durante el Clásico medio y tardío podría resumirse en un contexto cultural, en el cual las poblaciones de dicha región habían aceptado la identidad, de alguna forma, emanada de Teotihuacan, ya que muy probablemente la región entera había quedado incorporada al sistema político, económico y social que el gran estado había construido. A partir del hecho de que las manifestaciones tangibles de aquella urbe se reflejan en los procesos deliberados de imitar o reproducir los elementos culturales teotihuacanos, podría incluso conjeturarse que los habitantes de dicha región se identificaron como parte de la gran tradición de la llamada Ciudad de los Dioses. Éstas, como hemos mencionado de manera sucinta en párrafos anteriores, incluyen desde el nivel más básico y arraigado, que corresponde a las prácticas cotidianas, hasta la esfera simbólica que pertenece a las ideológicas y rituales. Entre los materiales más relevantes y ubicuos en la vida cotidiana se encuentran las piezas monocromas, por regla general, sin

\footnotetext{
25 Covarrubias, 2009.

26 Zepeda, 2009. Encastin, 2012.

27 Kabata, 2010. Jaimes, 2011.

28 Sugiura, et al., 2010.
} 
decoración, como ollas, cántaros, cazuelas, comales e incluso cajetes de servicio. Éstos muestran signos inequívocos de su filiación teotihuacana, sobre todo en sus características morfológicas, como las formas semi-esféricas o abiertas con o sin soportes anulares (figura 3). También se reconoce la gran similitud en el predominio de colores exteriores en variaciones de cafés, tonos más obscuros que los que caracterizan el color de la cerámica del siguiente período Epiclásico, así como en su técnica de elaboración con el moldeado o técnicas mixtas.

Entre las piezas cerámicas utilizadas para actividades no utilitarias que exhiben claras filiaciones teotihuacanas, destacan los vasos bruñidos monocromos ya sea de café obscuro o negruzco o rojo. Algunos de estos están profusamente decorados en la pared exterior, en donde sobresalen motivos, compuestos por elementos, a simple vista, identificables como teotihuacanos (figura 4).

También vale la pena mencionar los cajetes bicromos rojo sobre café, con o sin líneas incisas que delimitan los motivos decorativos. Numérica-

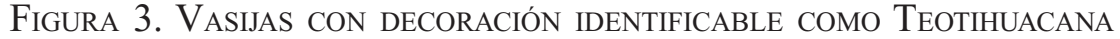

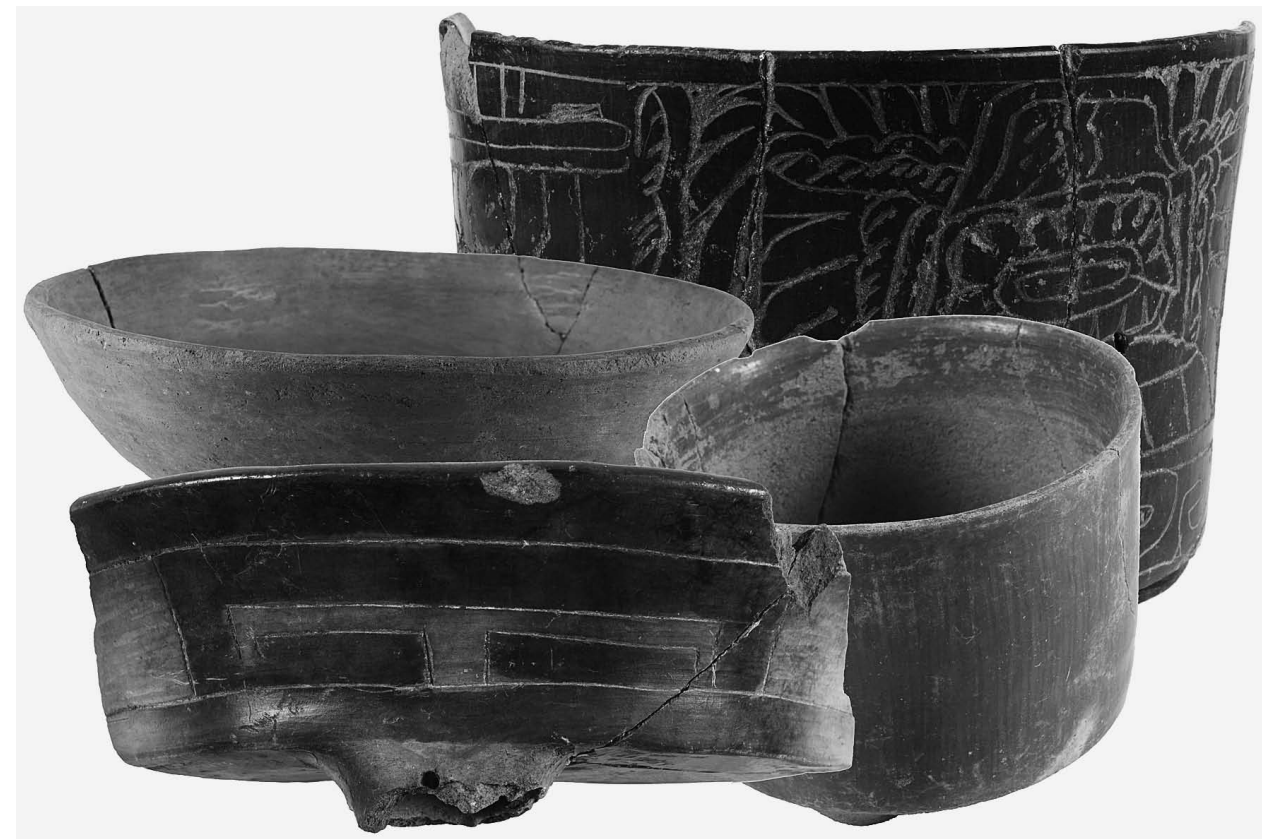

Fuente: elaboración propia. 
Figura 4. Formas CERÁmicAs CON ElEMENTOS ALUSIVOS A LA COSMOVISIÓN TEOTIHUACANA

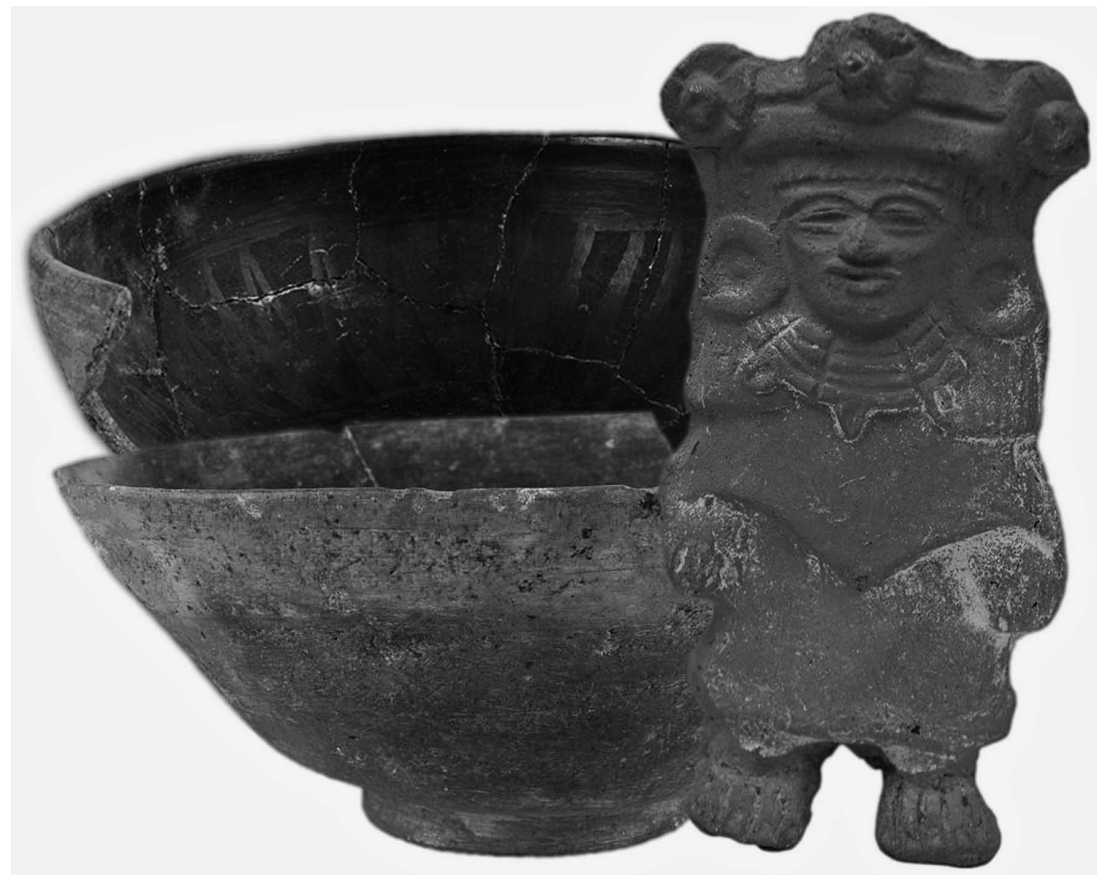

Fuente: elaboración propia.

mente hablando, éstos constituyen la minoría entre los materiales cerámicos, no obstante, su escasa presencia, aunada a la gran energía invertida en la elaboración de cada pieza y su alta similitud con los elementos genuinamente teotihuacanos, denotaría su importancia particular en la esfera de acciones alejadas de las prácticas cotidianas ${ }^{29}$. Desde la misma perspectiva, vale la pena mencionar otros objetos de barro que se consideran forman parte de la tradición teotihuacana. Se trata de floreros, sahumadores y braseros adornados con elementos alusivos a la cosmovisión teotihuacana, así como las figurillas hechas de molde con tocados, adornos e indumentaria que las identifican con la tradición de la Ciudad de los Dioses en su etapa final ${ }^{30}$.

\footnotetext{
29 Rattray, 2001.

30 Silis García, 2005.
} 
Distanciamiento al Estado teOtihuacano y GeStación DE LA IDENTIDAD EN EL VALLE DE TOLUCA

Como todo ámbito político y económico que representa un estado de magnitud como el de Teotihuacan, su desarrollo se caracteriza por procesos no sólo complejos, sino también contradictorios, en los cuales, desde antes del ocaso de la ciudad, ya se denotan los primeros síntomas de su declive ${ }^{31}$. Esta complejidad se manifiesta dentro del proceso mismo que la transformó en un estado panmesoamericano. Así, cuando Teotihuacan se encontraba en la cúspide, comenzaba ya a evidenciarse cierta contradicción y disfunción al interior de su sistema, la cual se percibe más notable al sur y oriente de la cuenca de México ${ }^{32}$.

Es también posible conjeturar que, conforme se agudizan los problemas al interior del estado hacia los finales de la fase Xolalpan, el aparato político teotihuacano intensificó su control sobre ciertas regiones como el caso del valle de Toluca, donde su mayor injerencia sobre sus poblaciones se aprecia en la creciente tendencia poblacional detectada a partir del Clásico medio al tardío, correspondiente a las fases teotihuacanas Tlamimilolpa tardío y Xolalpan tardío. El patrón de asentamiento sugiere que un gran número de población teotihuacana se dirigió hacia el valle de Toluca, por un lado, para ejercer un mayor control sobre la región y, por el otro, en busca de mejores condiciones de supervivencia. La aparición de algunos centros regionales estratégicamente ubicados con relación a la cuenca de México podría, también, considerarse como otra manifestación del mismo fenómeno ${ }^{33}$.

En este contexto, el papel que jugó el valle de Toluca merece una atención particular, dada la larga historia de relaciones establecidas entre dicha región y la gran urbe. Ciertamente el valle de Toluca carece de materias primas de carácter suntuario como serpentina, jadeíta, cristal de roca, travertinos, concha, entre otros, pero se ha conocido por la riqueza de los recursos naturales y por la alta productividad agrícola, sobre todo del maíz, grano básico que se requería para subsanar la exigencia alimenticia de la enorme población urban ${ }^{34}$. Probablemente éstos constituían materiales estratégicos para Teotihuacan, cuya obtención mediante aseguramiento de rutas de abastecimiento se consideró sine qua non para mantener su preeminencia. Seguramente, una parte considerable de todo el producto agrícola, algunos recursos lacustres y forestales

\footnotetext{
31 Hirth y Cyphers, 1988.

32 Nalda, 1997.

${ }^{33}$ Sugiura, Barba, et al., 2010. Sugiura, Zepeda, et al., 2010. Sugiura en prensa a.

34 Sugiura y McClung, 1988.
} 
de esta región, se debió haber canalizado hacia Teotihuacan, aprovechando su cercanía física, histórica y social. La importancia del valle de Toluca se denota, además, por su ubicación estratégica, por donde circulaban productos de diversas regiones, algunos de los cuales eran codiciados para legitimar su estatus quo. Tampoco se debe olvidar el factor social de raigambre otomiano, el cual constituía un núcleo importante tanto en la población teotihuacana como en la del valle de Toluca.

El patrón de asentamiento así como la cultura material arqueológicos son testimonios de la estrecha relación con el desarrollo de Teotihuacan, debido a que el valle de Toluca, como una región vital, incidió activamente en el proceso que le permitió a convertirse en un estado hegemónico y mantenerse como tal durante cientos de años. Suele suceder que, cuando un estado crece desproporcionadamente y padece cada día mayor contradicción en el seno de la sociedad misma, se acelera, por un lado, el proceso de descomposición y, por el otro puede detonarse, en el ámbito político, una estrategia del estado para asegurar su dominio sobre las regiones que formaban parte central de su sistema $^{35}$. Esto implicó que Teotihuacan fue obligado a depender, cada vez más, de regiones como la del valle de Toluca, donde abundaban los recursos vitales $\mathrm{y}$, al mismo tiempo, mantener el control sobre ellas para la sobrevivencia de un estado que creció de forma ininterrumpida por centurias.

Como se ha observado en la historia humana, el proceso de descomposición de un estado poderoso se traduce, con frecuencia, en la intensificación de injerencia por parte del poder hegemónico, en este caso de Teotihuacan, en la vida de los habitantes del valle de Toluca. Toda formación social, conforme adquiere mayor complejidad, se caracteriza por procesos cada vez más intricados, donde se conjugan intereses en diversas direcciones, frecuentemente contrarios e incompatibles. El contexto que caracteriza el proceso de desintegración del mundo teotihuacano no fue la excepción. Por un lado, se intensifica la política cohesiva del Estado hacia las regiones vitales para su sobrevivencia, por otro lado esta misma estrategia propicia la gestación de un proceso de alejamiento o rechazo hacia el otrora poder hegemónico, en donde los habitantes de aquellas regiones anteriormente habían aceptado formar parte del mismo, de manera voluntaria o forzada. Este proceso fortalece, a su vez, el sentido de pertenencia a la región local.

Bajo estas circunstancias, la condición que permitió la emergencia y mantenimiento de la identidad local, fue la creación de ciertos códigos o estilos que sirvieron como instrumentos para el reconocimiento de la población originaria, en este caso concreto, del valle de Toluca. La cultura material es, sin duda,

\footnotetext{
${ }^{35}$ Sugiura, en prensa b.
} 
emblemática para comprender este complejo proceso hacia la construcción identitaria. Naturalmente es importante reconocer que sus manifestaciones, por provenir de procesos intricados, no deben reducirse sólo a ella, pues la comprensión de la identidad está sujeta a fenómenos complejos que pueden quedar encubiertos por las similitudes en la cultura material ${ }^{36}$.

Retomando lo dicho anteriormente, se resume que la prueba más patente de la evidente incorporación de la región toluqueña al poderoso sistema teotihuacano se encuentra en el hecho de que las manifestaciones culturales en el valle de Toluca, ya sea patrón de asentamiento, estilo y orientación arquitectónicos, técnica de construcción, manufactura de cerámica y lítica o parafernalia ritual-ideológica, exhiben el alto grado de similitudes con la cultura teotihuacana, fenómeno que sólo se entiende, tomando en consideración la posible incorporación de la región al macro sistema teotihuacano. Entre diversos elementos que sustentan dicha conjetura, la cerámica ocupa un lugar privilegiado por sus cualidades particulares que manifiestan una gran sensibilidad a cambios sociales y políticos ${ }^{37}$. Prueba de ello es que la cerámica del valle de Toluca perteneció a la gran tradición teotihuacana, sin que esta situación cambiara radicalmente hasta el final del Clásico.

Si bien el valle de Toluca nunca se desligó, por completo, de su esfera política, conforme avanzó el proceso de descomposición, se fue gestando un proceso sutil de inconformidad frente al dominio teotihuacano, el cual fue cobrando cada vez mayor fuerza al acercarse el ocaso de la gran ciudad. Éste se aprecia en la aparición y pronta difusión de innovaciones que caracterizan algunos materiales cerámicos de fines del Clásico. Si bien son expresiones sutiles y difíciles de reconocer a primera vista, constituyen elementos que aparecen de manera recurrente, cubriendo a lo largo y ancho el valle de Toluca durante un lapso muy corto, pero de gran relevancia desde el punto de vista de los sucesos históricos. Se trata de una serie de objetos cerámicos, que presentan, por un lado, aparentes imitaciones de algunos elementos que manifiestan la gloria teotihuacana y, por el otro, modificaciones en los motivos decorativos, aunque ejecutados dentro de los cánones teotihuacanos en cuanto a formas y técnicas de elaboración.

En cuanto a la primera, cabe destacar el caso de la cerámica denominada como Pseudo-anaranjado delgado, cuya apariencia, tanto morfológica como de color exterior, recuerda de inmediato a la cerámica Anaranjado delgado, considerada como uno de los elementos indicativos del poder de dominio

${ }^{36}$ Insoll, 2007: 15.

37 Rattray, 2001. 
teotihuacano sobre gran parte de Mesoamérica ${ }^{38}$. El Pseudo-anaranjado delgado consiste fundamentalmente en cajetes abiertos sin decoración y con o sin soporte anular, salvo unas cuantas piezas decoradas con la técnica de punzonado. Su característica particular, que permiten reconocer inmediatamente su procedencia, consiste en un reborde en la parte superior de la pared exterior. Los colores de superficie pueden presentar irregularidades, variando considerablemente entre el anaranjado y negruzco, incluso con cierta tonalidad metálica o tornasolada. Las formas y colores exteriores más comunes de este grupo cerámico del valle de Toluca coinciden con los cajetes semiesféricos del Anaranjado delgado que se supone Teotihuacan controlaba su circularon, aunque cabe señalar que el material del valle de Toluca se distingue por su homogeneidad, careciendo la variabilidad registrada en Teotihuacan, sobre todo algunas piezas únicas con formas y decoraciones espectaculares.

Dado que esta cerámica se considera emblemática del poder que ostentó la gran urbe durante cientos de años, su producción, uso y amplia distribución en el valle de Toluca puede interpretarse como un testimonio de que los habitantes de dicha región requerían aún legitimarse frente al estado teotihuacano y ampararse bajo su tutela. Pero también, este fenómeno puede traducirse como una expresión de distanciamiento. Indudablemente, en la trama histórica compleja como la descomposición de un gran estado, un mismo objeto puede manifestar significados disímiles, incluso opuestos, como el caso de esta cerámica que remite un doble mensaje, es decir, la sujeción al mundo teotihuacano y su alejamiento.

Por su parte, hacia finales del Clásico, aparecen una serie de objetos cerámicos monocromos o bicromos que se caracterizan por su corta duración y su amplia distribución en la región toluqueña. Éstos, que evidencian esta doble identidad, expresan, a simple vista, una gran semejanza con los materiales genuinamente teotihuacanos, insinuando que forman parte de la tradición de la gran urbe. La semejanza con la cerámica teotihuacana se denota en sus técnicas de acabado a palillo que produce un alto lustre; en sus formas, como vasos, ollas de uso no utilitario y cajetes semiesféricos, además de sus técnicas decorativas como el esgrafiado y pulimento zonal o en patrón ${ }^{39}$.Todos estos materiales adoptan, en términos generales, cánones estéticos, así como técnicas de manufactura de la tradición teotihuacana, pero al mismo tiempo, difieren de los elaborados en aquella ciudad. Entre los materiales más representativos, se destaca el caso de los vasos bruñidos de poca profundidad, los cuales frecuentemente tienen decoración esgrafiada en el exterior, técnica que

\footnotetext{
38 Encastin, 2012.

39 Rattray, 2001.
} 
se caracteriza la tradición de la gran urbe. Si bien estas piezas pueden considerarse parte del legado cultural de Teotihuacan, se identifica, a simple vista, su procedencia toluqueña por la disposición de motivos sencillos, distintivos de la región de Toluca ${ }^{40}$ (ver arriba figura 3 y 4).

La misma técnica de esgrafiado se encuentra, también, en algunas ollas bruñidas, sin huellas aparentes de haber sido sometida al fuego ni de haberse utilizado para usos cotidianos. Su decoración se caracteriza por motivos recurrentes, los cuales consisten principalmente de figuras cursivas geométricas, combinadas con decoración de pulimento zonal, técnica que es característica de la tradición de las fases tardías de Teotihuacan. Estas ollas de tamaño pequeño o mediano se distinguen, por regla general, por un cuerpo globular, cuello alargado y borde evertido. A diferencia de las ollas utilitarias, no tienen asas y la base plana, con o sin soportes trípodes de botón. A pesar de la amplia distribución dentro del valle de Toluca, su presencia comprende exclusivamente un lapso muy corto definido entre las fases de Atizapán y Tejalpa del valle de Toluca o Xolalpan y Metepec de Teotihuacan.

Un ejemplo que pertenece a este grupo cerámico el cual, junto con la cerámica esgrafiada, se encuentra distribuido ampliamente en el valle de Toluca se refiere a los cajetes abiertos con o sin soporte anular, decorados con los motivos hechos por la técnica de «pulimento a palillo», diagnóstico de la cerámica teotihuacana de las fases Xolalpan y Metepec. El sello distintivo de este grupo cerámico llamado «pulimento en patrón» del valle de Toluca consiste en los motivos decorativos, los cuales suelen ubicarse en el interior de cajetes en posición contrapuesta, formando figuras geométricas ya sea de círculos concéntricos o líneas rectas cruzadas o paralelas, así como motivos cursivos ${ }^{41}$. Así mismo, la gran homogeneidad morfológica de los cajetes semiesféricos debe considerarse como una expresión propia de la región.

La aparición y rápida difusión de estas modificaciones cerámicas, sutiles pero consistentes, coexistentes con otros elementos culturales que aún manifiestan una clara adhesión a los cánones teotihuacanos, parecen indicar que, hacia los finales del Clásico, se fue gestando un proceso complejo de identidades, por un lado, la regional que debe considerarse como una herramienta para la autodefinición del valle de Toluca y, por el otro, la identidad asumida o, en cierta forma, impuesta como parte del mundo teotihuacano que funcionó hasta el final de este gran estado mesoamericano.

\footnotetext{
${ }^{40}$ Idem.

41 Zepeda, 2009.
} 


\section{Consolidación de LA IDENTIDAD Regional DEL VAlle DE TOlucA}

La tendencia de continuo crecimiento del número de sitios en el valle de Toluca se aprecia aun cuando el proceso de decline teotihuacano se manifestaba de manera irreversible durante la fase Metepec (550-600 dC), cuando ocurrió el virtual ocaso de la gran metrópoli mesoamericana (Sugiura 2005a). Durante este tiempo, en el cual el valle de Toluca aún conservaba la identidad de pertenecer a la esfera teotihuacana.

En efecto, la desintegración del sistema teotihuacano, cuyo control directo o indirecto se extendió en gran parte de Mesoamérica dejó huellas profundas en todos los ámbitos de la vida de los pueblos del valle de Toluca ${ }^{42}$. El confuso tiempo, inmediatamente antes y después de la caída de Teotihuacan, se refleja en la cultura material, sobre todo en la cerámica, de la fase llamada Tejalpa (ca.600-650 dC), en la cual coexisten elementos propios del Clásico teotihuacano y que conformarán los del siguiente periodo, el Epiclásico. Éste (650-900 dC) como toda etapa de transición se caracteriza por su gran complejidad en diversos ámbitos ya sea social, político, económico y cultural (figura 5).

En el caso del valle de Toluca, el ocaso de Teotihuacan repercutió en forma positiva y propició el súbito crecimiento poblacional, consecuencia de la continua llegada de inmigrantes a sus fértiles tierras. Para esas fechas, la región alcanza la primera etapa de apogeo en su historia. El patrón de asentamiento evidencia una clara tendencia hacia una mayor complejidad social, la cual se manifiesta no sólo en el súbito incremento del número de sitios, sino también en la clara diversidad de funciones al interior de ellos. La aparición de algunos centros de magnitud y complejidad que hasta entonces no se habían conocido como son los casos de Teotenango y Techuchulco puede interpretarse dentro de este contexto histórico. El proceso de consolidación del valle de Toluca como una región con su propia identidad se aprecia, también, en el crecimiento de otros que se fundaron en tiempos anteriores como Santa Cruz Atizapán. Éstos y otros centros regionales con un número considerable de asentamientos aledaños formaban unidades políticas, relativamente pequeñas, que controlaban flujos de los recursos regionales, así como las rutas de intercambio entre la tierra caliente y el valle de México.

El fenómeno epiclásico apunta el inicio de una nueva etapa, que rompe con el esquema histórico previo y que, si bien las huellas de la tradición de aquella gran urbe no se borraron por completo, viene a reemplazar el legado

${ }^{42}$ González de la Vara, 1998. Sugiura, 2006. Sugiura, Barba, et al., 2010; Sugiura, Zepeda, et al., 2010. Sugiura, 2011. 
Figura 5. Patrón de asentamiento Epiclásico

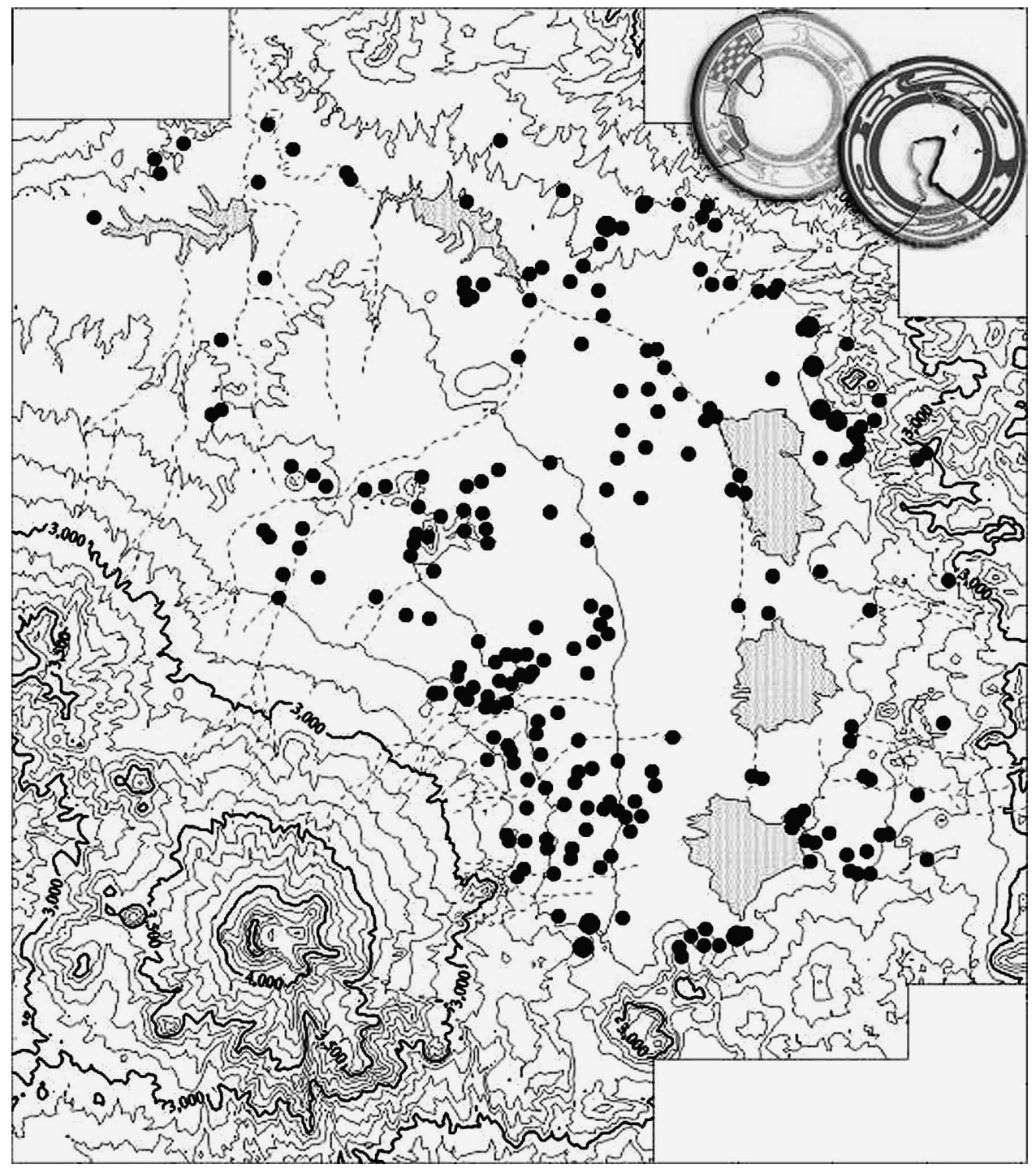

Fuente: elaboración propia.

cultural teotihuacano. Entre los cambios más relevantes, se destacan, por un lado, la aparición de nuevos elementos culturales como la arquitectura pública de planta circular, pero, sobre todo, la cerámica conocida como Coyotlatelco 
con su estilo decorativo característico de rojo sobre bayo (figura 6). También, se debe tomar en consideración nuevas esferas de interacción e intercambio con regiones hacia el occidente mesoamericano y el sur del actual Estado de México, las cuales se manifiestan por la presencia de obsidiana de Ucareo, Michoacán y una serie de objetos cerámicos provenientes de la tierra caliente, al sur del Estado de México. Por el otro lado, desaparecen el uso de pizarra en contextos rituales y algunas formas cerámicas, las cuales tienen implicaciones en el ámbito ideológico teotihuacano como candeleros, floreros, vasos esgrafiados con motivos simbólicos. También dejan de circular piezas de piedra verde y obsidiana verde de Pachuca, Hidalgo. Naturalmente, el uso de elementos culturales que jugó un papel fundamental para mantener Teotihuacan su preeminencia en una vasta región mesoamericana no dejó de funcionar, por completo, aún después de su caída. Prueba de ello es la pervivencia, naturalmente con ciertas modificaciones, de algunos elementos con cierta connotación ideológica, creada por Teotihuacan, siguió utilizándose aún después de su

\section{Figura 6. Cerámica Coyotlatelco}

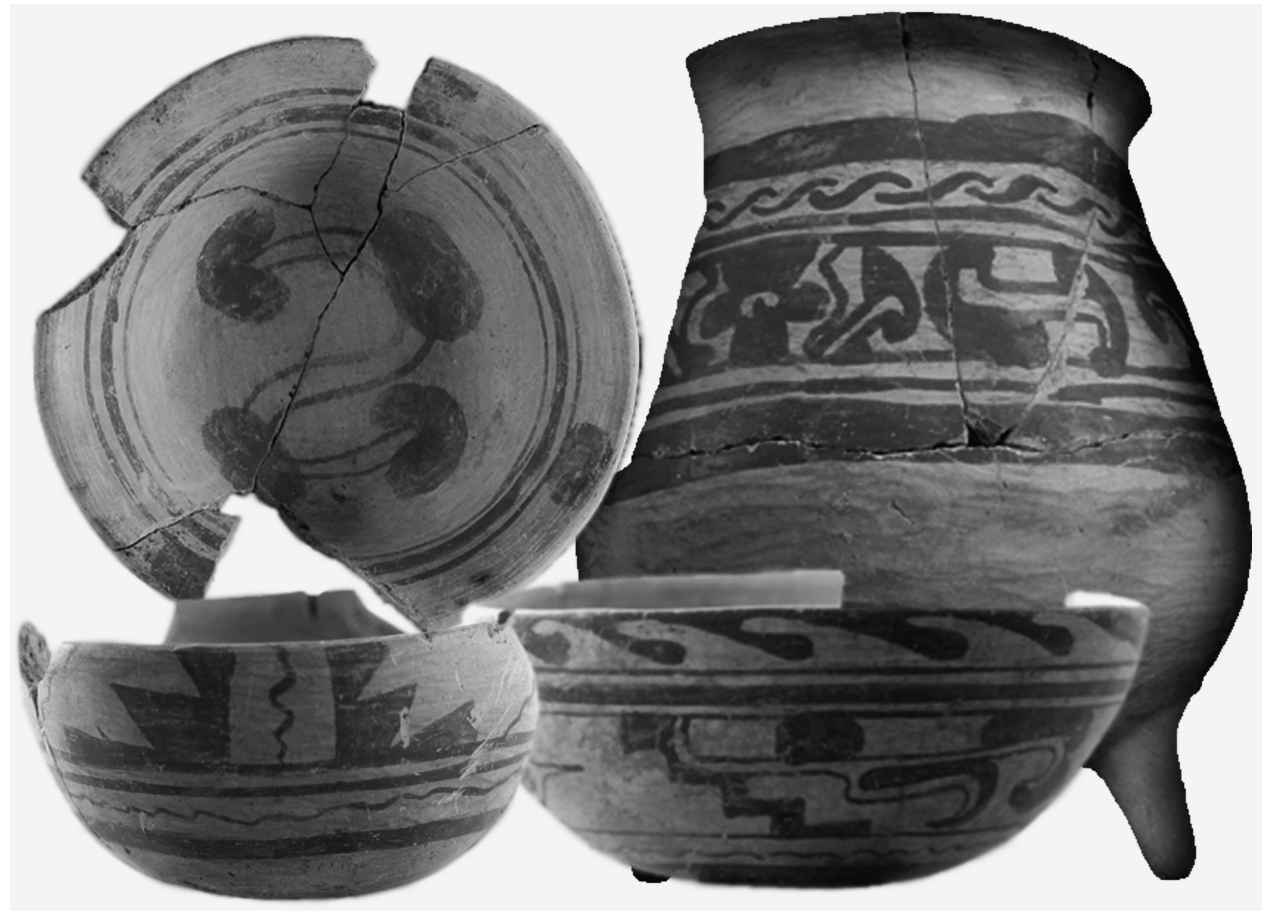

Fuente: elaboración propia. 
ocaso como se observan en los motivos decorativos de adornos de incensarios y braseros, así como figurillas, elemento arquitectónico de talud y tablero.

Entre la cultura material, sin duda, la cerámica ha jugado un papel preponderante en una gran variedad de temas acerca de las sociedades pretéritas. Las ventajas que posee este material arqueológico, que constituye una de las variables más sensibles para acercarnos al tema del presente estudio, se atribuye precisamente a sus cualidades particulares ampliamente reconocidas en la arqueología ${ }^{43}$. Prueba de ello es que la cerámica Coyotlatelco ha jugado el papel de indicador más representativo en la compleja trama histórica a raíz del ocaso de Teotihuacan.

Independientemente de los debates que existen acerca de su origen u orígenes, el complejo Coyotlatelco ${ }^{44}$ que viene a reemplazar la tradición cerámica teotihuacana anuncia un nuevo orden histórico, cuyo desarrollo pleno se ubica en el Posclásico hacia $900 \mathrm{dC}$. Los datos arqueológicos provenientes del valle de Toluca sugieren que dicha región pudo haber jugado un papel central en el desarrollo de este complejo cerámico que, al comparar con los materiales teotihuacanos, se distinguen por una serie de rasgos: la menor diversidad morfológica y la menor variación en las técnicas, motivos y colores decorativos. El Coyotlatelco consiste principalmente en la aplicación de engobe rojo sobre color natural del barro y, en casos limitados, el blanco sobre rojo, además de las decoraciones negativas, incisos y sellados. A diferencia de la cerámica teotihuacana, la producción del Coyotlatelco no estuvo centralizada en un centro productor, sino más bien consistió en varias localidades de escala limitada.

Por su parte, algunas esferas económicas que estableció Teotihuacan dejaron de funcionar, en su lugar se crean nuevas redes de interacción. Al deslindarse de la tutela de la gran urbe, el valle de Toluca estableció nuevos vínculos. A través de estas redes, se fluye una serie de nuevos objetos como las cerámicas provenientes de las tierras calientes al sur de la región (la llamada Engobe Naranja Grueso, Mica foránea y Rojo foráneo) y la lítica, principalmente, de Ucareo, Michoacán. En el ámbito doméstico, el inventario cerámico parece insinuar cambios en las prácticas cotidianas, manifiestos en la mayor popularización del uso de «comal», mientras que, en el nivel ideológico, pervive el uso de representaciones simbólicas del tiempo anterior.

De esta manera, el proceso hacia una identidad propia del valle de Toluca se ve fortalecido a raíz del ocaso de Teotihuacan, aunque el camino no representa una dirección linear, sino con tramas mucho más complejas, donde, junto con los nuevos elementos, siguen apareciendo algunos de tiempos anteriores.

43 Shepard, 1956. Rice, 1987. Sugiura, 2001.

44 Tozzer, 1921. Rattray, 1965. Sugiura, 2005a; 2006; en prensa a. 
Alrededor de $900 \mathrm{dC}$., el Coyotaltelco deja de tener importancia dentro del panorama cultural del valle de Toluca. En su lugar, coincidiendo con el surgimiento del grupo hegemónico conocido como Matlatzinca, aparece un nuevo complejo cerámico identificado con el mismo nombre (figura 7). Durante esta nueva etapa histórica mesoamericana denominada Posclásico, se detecta que el número de sitios en el valle de Toluca siguió en aumento y que se colonizaron nuevas zonas dentro de la región. En este contexto, algunos centros regionales de escala moderada siguieron creciendo, incorporando otros asentamientos cercanos y convirtiéndose en centros de considerable magnitud como Calixtlahuaca ${ }^{45}$, Teotenango ${ }^{46}$, Techuchulco y Santa Cruz Atizapán ${ }^{47}$. La

\section{Figura 7. Cerámica Matlatzinca}

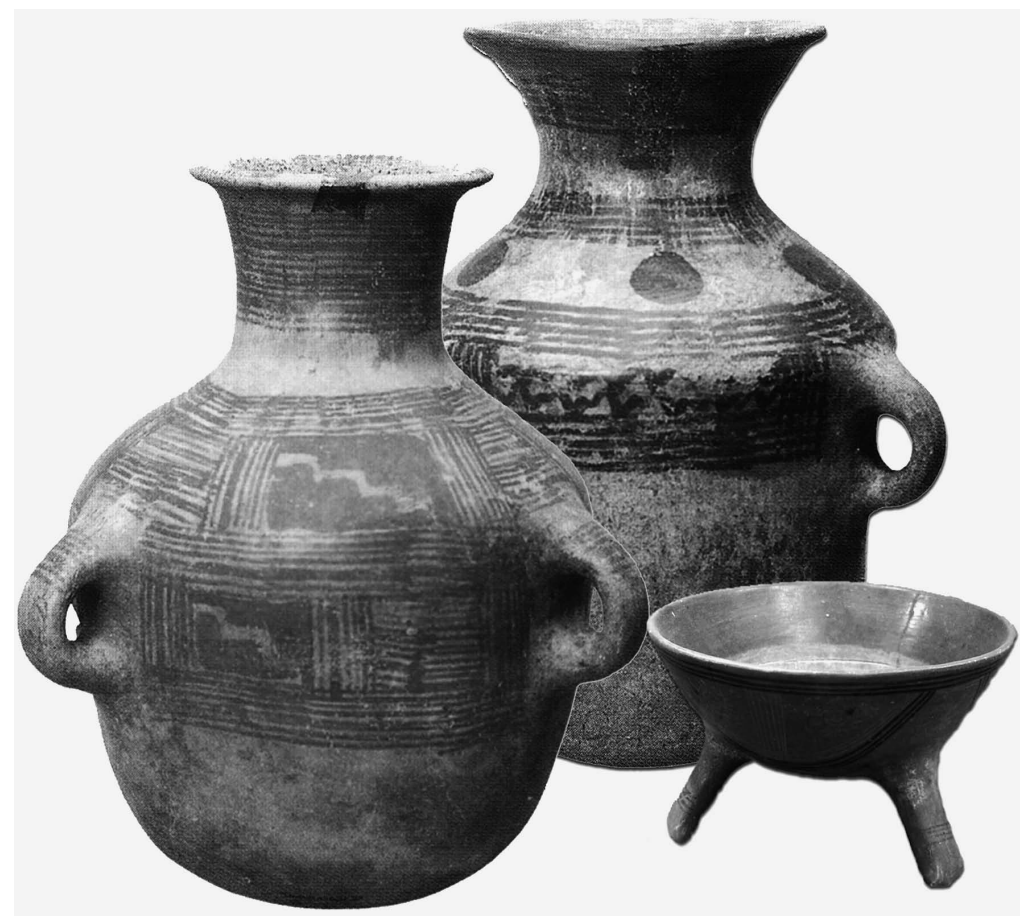

Fuente: elaboración propia.

${ }^{45}$ García Payón, 1936. Smith, et al., 2003. Nieto, 2007. Smith, et al., 2007; 2009. Nieto, 2008. Tomaszewski y Smith, 2011.

${ }^{46}$ Piña Chan, 1975.

47 Sugiura, 2011. 
historia posclásica del valle de Toluca refleja, por un lado, una complejidad social y política que hasta entonces no se ha conocido y, por el otro, la plena consolidación de identidades étnicas, las cuales se manifiestan en una serie de cultura material desde el patrón de asentamiento, la casa habitación, forma de llevar a cabo prácticas cotidianas, incluso en su base artefactual hasta el uso de símbolos particulares en actos rituales y de ceremonias.

La diversidad regional que se refleja en la cultura material se distingue, sobre todo, en la cerámica. Así, el complejo Matlatzinca carece del uso de «comal» para calentar las «tortillas», una forma cerámica cuya presencia ya se había identificado desde tiempos atrás entre los menajes cotidianos del valle de Toluca. No parece haber utilizado figurillas de barro para propósitos ceremoniales, en cambio se han manufacturado cajetes de servicio que presentan alta calidad de acabado y, frecuentemente, decorados en rojo o en policromía. A diferencia de los matlatzincas, el grupo otomí utilizaba los comales, los cuales tienen una forma distintiva que no comparte con las de otros comales identificados en tiempos anteriores. Otra característica de este grupo es, visto morfológicamente, se conforma por un número muy reducido de formas cerámicas, consistidas tan sólo en cuatro formas principales: ollas, cuencos, «tecomates» y comales, careciendo de formas como cajetes de servicio que representan el material matlatzinca. Esta diversidad detectada en el material cerámico parece coincidir claramente con la distribución de sitios, indicando las fronteras étnicas dentro de la región.

En resumidas cuentas, el crecimiento poblacional, patrón de distribución de sitios arqueológicos, desarrollo de centros importantes con dominio geopolítico de mayor escala y el regionalismo cultural manifiesto en la distribución de diversos complejos cerámicos, además del Matlatzinca, parecieran estar relacionados con la gestación y consolidación de diversos grupos étnicos de raigambre otomiano que anteriormente sólo se identificaba como tal, sin conformar identidades más específicas como Matlatzinca, Otomi y Mazahua ${ }^{48}$. En efecto, no sería correcto decir de la identidad regional del valle de Toluca, dado que el panorama social se ha vuelto mucho más complejo con la consolidación de grupos etno-lingüísticos al interior de la región.

En este proceso, la lucha por el control de la riqueza ambiental jugó un papel determinante. En el registro arqueológico se observan relaciones desiguales entre los grupos poblacionales asentados en diferentes zonas ecológicas con acceso a recursos diferenciados. Prueba de ello es que los Matlatzinca que ocuparon las zonas más fértiles del valle se convirtieron en el grupo dominante, mientras que los otomíes fueron relegados hacia los lugares marginales.

${ }^{48}$ Sugiura, 2005a. 
Hacia la segunda mitad del siglo XV, el valle de Toluca queda sometido bajo el dominio de la Triple Alianza, con el que se introduce el náhuatl como la lengua franca ${ }^{49}$. Posteriormente este escenario multiétnico se ve frenado por la invasión europea en el siglo XVI.

Si bien la cultura material en su conjunto constituye una base relevante $\mathrm{y}$, en efecto, se ha utilizado por diversas corrientes teóricas para identificar el proceso de cambio, la cerámica arqueológica, sobre todo en las sociedades prehispánicas de Mesoamérica, ha sido singularizada como un elemento que denota de forma más sensible las transformaciones sociales. Con base en lo anterior, es posible conjeturar que los estudios de materiales cerámicos nos permiten adentrarnos en la esfera identitaria ya sea local o regional. El caso de la región bajo estudio no es una excepción.

\section{Consideraciones finAles}

En el presente estudio hemos discutido que, como toda manifestación social, la identidad es también un proceso multidimensional. Quizá las polémicas y discrepancias teóricas que existen entorno a dicha noción se atribuyen precisamente a esta complejidad y a diferentes posturas que abordan el tema ya sea de las sociedades contemporáneas o, como este caso concreto, pretéritas. Lo que es innegable es el hecho de que las categorías aisladas a través de las cuales se trató la identidad en la primera parte del siglo XX, quedaron cortas para comprender este proceso. Es, también, incuestionable la riqueza que podemos alcanzar desde una perspectiva integral, que resalta la pertinencia de tomar en consideración los contextos particulares de caso, para la cual no se concibe la identidad como una cualidad adscrita ni una condición definida o asignada a priori.

A partir de lo anterior, hemos dejado en claro la razón y pertinencia de concebir la identidad como un proceso dinámico, constreñido por su contexto histórico y las condiciones materiales de tiempo y espacio, que como tal influye en el grupo como colectividad. Por su parte, hemos enfatizado que la identidad permite a los grupos sociales asumir un sentido de pertenencia como parte de un «nosotros» que denota, a su vez, la distinción o diferenciación con respecto a otros. En el presente estudio nos referimos a una identidad colectiva, concretamente hablando de las antiguas poblaciones del valle de Toluca, que sintetiza y delimita las aspiraciones del grupo mediante estrategias de negociación que invariablemente afectan las relaciones que establecen entre sí.

49 Piña Chan, 1975. Sugiura, 2005a; 2011. 
Esta perspectiva nos ha permitido aproximarnos oportunamente a la intricada trama del proceso de identidad en la región del valle de Toluca, que estuvo incorporada al gran sistema teotihuacano durante siglos. Una manifestación de este dominio se expresa por las pautas culturales impuestas por el gran estado pan-mesoamericano, es decir de «ellos», que los toluqueños adoptaron como una estrategia de sobrevivencia. A lo largo del texto, hemos señalado que esta relación entre Teotihuacan y el valle de Toluca fue adquiriendo un matiz diferente con el paso del tiempo. Hacia finales del Clásico, la aparición de los primeros síntomas de decline, seguida por el ocaso de Teotihuacan, repercutieron, de manera notable en la historia del valle de Toluca, puesto que los cambios y transformaciones surgidos en cualquier ámbito de la sociedad generan una respuesta, ya sea de adopción o rechazo, pero que afecta la forma, duradera o transitoria, en que los individuos se relacionan entre sí.

Este proceso tuvo como consecuencia en la región el surgimiento de la necesidad de negociar su identidad frente al poder bajo cuyo dominio se mantuvo durante siglos, manifestando cierto distanciamiento hacia el mismo, a la vez que se autoafirmó como una región con características propias. Así, se fue propiciando un incipiente proceso de gestación hacia una identidad colectiva de la población con respecto a la región a la cual pertenece, es decir de un «nosotros».

Hemos abordado este complejo proceso de negociación constante entre «nosotros»y «ellos» desde la perspectiva de una serie de materiales cerámicos pertenecientes al Clásico tardío y terminal, por ser considerados como un indicador con gran potencial interpretativo. Estos se caracterizaban por elementos diagnósticos, tanto morfológicos como de técnicas decorativas, que formaban parte de la tradición inconfundiblemente teotihuacana. Conforme transcurrió el tiempo, comenzó la aparición de vajillas cerámicas que si bien exhiben características propias de la tradición de la gran urbe, denotan rasgos o modificaciones que, a pesar de ser discretos, anuncian ya los indicios de una identidad propia de la región, como son los casos del llamado pseudoanaranjado delgado, el grupo cerámico con patrón de pulimento, así como la cerámica esgrafiada. La distribución de estos materiales en toda la región durante un lapso muy corto, pero significativo, el cual coincide con la fase terminal del otrora poderoso estado teotihuacano, y que se evidencia aún más claramente con los materiales cerámicos Coyotlatelco y Matlatzinca, que en su conjunto deben entenderse como un signo inconfundible de la necesidad de exaltar o reafirmar la identidad local de la población del valle de Toluca.

Sin duda, el desarrollo y proceso que conllevó a la desarticulación del poder teotihuacano tuvieron un camino sinuoso y complejo, cuyo análisis rebasa el presente artículo. Lo importante es resaltar que el proceso de desintegración 
no es sinónimo necesariamente del debilitamiento del poder ejercido en otras poblaciones fuera de la metrópoli, sino que frecuentemente propicia un efecto contrario. Como suele suceder en el plano político, conforme se acerca a su ocaso, la injerencia hacia las regiones satelitales que formaban parte de su sistema, como el caso del valle de Toluca, pudo haberse intensificado, dado que era apremiante obtener los productos necesarios para mantener no sólo su aparato político, sino también a la gran población urbana que se incrementaba con el paso del tiempo. Este momento crítico de gran complejidad propició múltiples consecuencias, entre las cuales destaca la gestación de un proceso incipiente de autoafirmación colectiva hacia la región perteneciente y de una tendencia hacia el distanciamiento del mundo del que formaba parte.

\section{BiBLIOGRAFÍA}

Anderson, Benedict, Imagined Communities. Reflections on the Origin and Spread of Nationalism, London, Verso, 1991.

Anderson, Margaret y Reeves, Andrew, "Contested Identities: Museums and the Nation in Australian", F. E. S. Kaplan, Museums and the Making of 'Ourselves': The Role of Objects in National Identity, Leicester, Leicester University Press, 1994: 79-124.

Bartra, Roger, La Jaula de la Melancolía: Identidad y Metamorfosis del Mexicano, México, Grijalbo, 1987.

Binford, Lewis R., In Pursuit of the Past, London, New York, Thames and Hudson, 1983.

Binford, Sally R. y Binford, Lewis R., New Perspectives in Archaeology, Chicago, New York, Aldine, Atherton, 1968.

Buentello, Leonor; Sugiura, Yoko y Pérez, Aída, "Tras las huellas genéticas de los isleños de Santa Cruz Atizapán", Yoko Sugiura, La gente de la ciénaga en tiempos antiguos. La historia de Santa Cruz Atizapán, México, UNAM/El Colegio Mexiquense, 2009: 127-144.

Cerulo, Karen A., "Identity Construction: New Issues, New Directions", Annual Review of Sociology, 23 (1997): 385-409.

Chorba, Carrie C., Mexico, from Mestizo to Multicultural. National Identity and Recent Representations of the Conquest, USA, Vanderbilt University Press, 2007.

Clark, Bonnie J., "Lived Ethnicity: Archaeology and Identity in Mexicano America", World Archaeology, 37/3 (2005): 440-452.

Covarrubias, Mariana, "El sistema constructivo de Santa Cruz Atizapán a través de los siglos", Yoko Sugiura, La gente de la ciénaga en tiempos antiguos. La historia de Santa Cruz Atizapán, México, UNAM/El Colegio Mexiquense, 2009: 145-162. 
Cowgill, George, “An update on Teotihuacan”, Antiquity, 82 (2008): 962-975.

Díaz-Andreu, Margarita; Lucy, Sam; Babic, Stasa y Edwards, David., The Archaeology of Identity. Approaches to gender, age, status, ethnicity and religion, London, Routledge, 2005.

Doremus, Anne "Indigenism, Mestizaje, and National Identity in Mexico during the 1940s and the 1950s", Mexican Studies / Estudios Mexicanos, 17/2 (2001): 375402.

Dresser, Madge, "Britannia", Raphael Samuel, Patriotism. The Making and Unmaking of British National Identity, London, Routledge, 1989: 26-49.

Du Gay, Paul; Evans, Jessica y Redman, Peter (eds.) Identity: a reader, London, SAGE in association with the Open University, 2000.

Encastin, Carolen, "La cerámica Pseudo anaranjado delgado como un indicador local, evidencia del vínculo entre Teotihuacan y el valle de Toluca en el Clásico (150-600/700dC)", Tesis de Licenciatura, Universidad Autónoma del Estado de México, 2012.

Escolar, Marcelo; Quintero Palacios, Silvina y Reboratti, Carlos, "Geographical identity and patriotic representation in Argentina", David Hooson, Geography and National Identity, Oxford, Blackwell, The Institute of British Geographers, 1994: 346-366.

Fleury-Ilett, B., "The identity of France: the archaeological interaction", Journal of European Archaeology, 1 (1993): 169-180.

Foote, Kenneth; Tóth, Attila y Árvay, Anett, "Hungary after 1989: Inscribing a New Past on Place", Geographical Review, 90/3 (2000): 301-334.

Fowler, Chris, "Personhood and Social Relations in the British Neolithic with a Study from the Isle of Man", Journal of Material Culture, 6/2 (2001): 137-163.

Gamio, Manuel, La Población del Valle de Teotihuacán, Mexico City, Dirección de Talleres Gráficos, 1922.

Gándara, Manuel, “La Vieja «Nueva Arqueología»: Segunda Parte”, Boletín de Antropología Americana, 3 (1981): 7-70.

García Payón, José, La zona arqueológica de Tecaxic-Calixtlahuaca y los matlatzincas: etnología y arqueología (primera parte), México, Talleres Gráficos de la Nación, 1936.

González de la Vara, Fernán, "Historia prehispánica del valle de Toluca”, Yoko Sugiura, Historia General del Estado de México: Arqueología, Tomo I, Toluca, Gobierno del Estado de México y El Colegio Mexiquense, 1998: 163-198.

González de la Vara, Fernán, El Valle de Toluca Hasta la Caída de Teotihuacan, México, INAH, 1999. 
González de la Vara, Fernán, "Historia prehispánica del valle de Toluca”, Yoko Sugiura, Historia General Ilustrada del Estado de México. Volumen I: Geografía y Arqueología, Toluca, El Colegio Mexiquense, 2011, I: 181-215.

Gosden, Chris, "Postcolonial Archaeology. Issues of Culture, Identity and Knowledge", I. Hodder, Archaeological Theory Today, Cambridge, Polity, 2001: 241-261.

Graham, Brian J., "The Search for the Common Ground: Estyn Evans's Ireland", Transactions of the Institute of British Geographers, 19/2 (1994): 183-201.

Hammond, Allen L., "The New Archaeology: Toward a Social Science”, Science, New Series, 172/3988 (1971): 1119-1120.

Hirth, Kenneth y Cyphers, Ann, Tiempo y asentamiento en Xochicalco, México, IIA/ UNAM, 1988. (Serie Monográfica, I).

Hodder, Ian, The present past: an introduction to anthropology for archaeologists, London, Batsford, 1982a.

Hodder, Ian, Symbols in Action: Ethnoarchaeological Studies of Material Culture, Cambridge, Cambridge University Press, $1982 \mathrm{~b}$.

Hodder, Ian, Reading the past: current approaches to interpretation in archaeology, Cambridge, Cambridge University Press, 1986.

Hodder, Ian, The archaeology of contextual meanings, Cambridge, Cambridge University Press, 1987.

Hodder, Ian, Archaeological theory in Europe: the last three decades, London, Routledge, 1991a.

Hodder, Ian, The meanings of things: material culture and symbolic expression, London, Harper Collins Academic, 1991 b.

Hodder, Ian, "The past as passion and play: Çatalhöyük as a site of conflict in the construction of multiple pasts", Lynn Meskell, Archaeology under fire. Nationalism, politics and heritage in the Eastern Mediterranean and Middle East, London, Routledge, 1998: 124-139.

Hodder, Ian, The archaeological process: an introduction, Oxford, Blackwell, 1999.

Hodder, Ian; Shanks, Michael; Alexandri, Alexandra; Buchli, Victor; Carman, John; Last, Jonathan y Lucas, Gavin, Interpreting archaeology: finding meaning in the past, London, Routledge, 1995.

Holtorf, Cornelius, "Notes on the Life History of a Pot Sherd", Journal of Material Culture, 7/1 (2002): 49-71.

Hooson, David (ed.), Geography and National Identity, Oxford, Blackwell. The Institute of British Geographers, 1994. Special Publications Series, 29.

Huddy, Leonie, "From Social to Political Identity: A Critical Examination of Social Identity Theory", Political Psychology, 22/1 (2001): 127-156. 
Hutchinson, John and Smith, Anthony D. (eds.), Nationalism, Oxford Readers, Oxford, Oxford University Press, 1994.

Insoll, Timothy, "Introduction: Configuring Identities in Archaeology", Timothy Insoll, The Archaeology of Identities: a Reader, London, Routledge, 2007: 1-18.

Jaimes, Gustavo, "La industria de obsidiana de San Mateo Atenco vista desde una perspectiva ecológica”, Tesis para obtener el grado de Licenciado en Arqueología, Inédita, UAEM, 2011.

Jenkins, Richard, Social Identity, London, Routledge, 1996.

Jones, Sian, "Discourses of Identity in the Interpretation of the Past", Paul GravesBrown, Sian Jones y Clive Gamble, Cultural Identity and Archaeology. The Construction of European Communities, London, Routledge, 1996: 62-80.

Jones, Sian y Graves-Brown, Paul, "Introduction: Archaeology and Cultural Identity in Europe", Paul Graves-Brown, Sian Jones y Clive Gamble, Cultural Identity and Archaeology. The Construction of European Communities, London, Routledge, 1996: 1-24.

Kabata, Shigeru, "La dinámica regional del intercambio: el valle de Toluca antes y después de la caída de Teotihuacan”, Tesis doctoral en Arqueología, Instituto de Investigaciones Antropológicas/Universidad Nacional Autónoma de México, 2010.

Kane, Susan, "The Politics of Archaeology and Identity in a Global Context", Susan Kane, The Politics of Archaeology and Identity in a Global Context, Boston, MA, Archaeological Institute of America, 2003: 1-10.

Kaya, Ilhan, "Identity and Space: The Case of Turkish Americans", Geographical Review, 95/3 (2005): 425-440.

Llobera, Josep, "Catalan national identity: the dialectics of past and present", Elisabeth Tonkin, Maryon McDonald y Malcolm Chapman, History and Ethnicity, London, ASA, 1989: 247-261.

Lyman, R. Lee; O'Brien, Michael J. y Dunnell, Robert, The Rise and Fall of Culture History, New York, Plenum, 1997.

Manzanilla, Linda, "The emergence of complex urban societies in Central Mexico: the case of Teotihuacan", Gustavo Politis y Benjamin Alberti, Archaeology in Latin America, London, Routledge, 1999: 93-129.

Meskell, Lynn, “Archaeologies of Identity”, Ian Hodder, Archaeological Theory Today, Cambridge, Polity, 2001: 187-213.

Meskell, Lynn, "The Intersections of Identity and Politics in Archaeology", Annual Review of Anthropology, 31 (2002): 279-301.

Millon, Rene, "The Teotihuacan Mapping Project", American Antiquity, 29/3 (1964): 345-352. 
Nalda, Enrique, "El noreste de Morelos y la desestabilización teotihuacana", Arqueología, 18 (1997): 103-117.

Nieto, Rubén; Sugiura, Yoko y Ricardo Jaramillo, "Panorámica arqueológica del valle de Malinalco", Xavier Noguez, Malinalco y sus contornos a través de los tiempos, Toluca, UAEM/El Colegio Mexiquense AC, 2006: 35-43.

Nieto, Rubén, "Paisaje e identidad entre los matlatzincas: Una aproximación”, Ponencia sin publicar presentada en la XXVIII Mesa Redonda de la Sociedad Mexicana de Antropología, México, 2007.

Nieto, Rubén, “Caracterización de cerámica matlatzinca del valle de Toluca: avances para el reconocimiento de un marcador de filiación étnica", Ponencia sin publicar presentada en el II Simposio de Arqueología. Arqueología de la identidad. Tenancingo, México, 2008.

Parsons, Jeffrey y Sugiura, Yoko, "Teotihuacan and the Epiclasic in Central Mexico", Deborah Nichols, y Christopher Pool, The Oxford Handbook of Mesoamerican Archaeology, Oxford, Oxford University Press, 2012: 309-323.

Piña Chan, Román, Teotenango: el antiguo lugar de la muralla. Memorias de las excavaciones arqueológicas T I y II, Toluca, Dirección de Turismo/Gobierno del Estado de México, 1975.

Rattray, Evelyn Childs, "An archeological and stylistic study of Coyotlatelco pottery", Thesis University of the Americas, 1965.

Rattray, Evelyn, Teotihuacan: Ceramics, Chronology and Cultural Trends, México University of Pittsburgh/INAH, 2001.

Rice, Prudence M., Pottery analysis: A Source Book, Chicago, University of Chicago Press, 1987

Sabloff, Paula L. W., Conversations with Lew Binford. Drafting the New Archaeology, Norman, University of Oklahoma Press, 1998.

Samuel, Raphael (ed.), Patriotism. The Making and Unmaking of British National Identity, London, Routledge, 1989.

Schiffer, Michael, Behavioural Archaeology, New York, Academic Press, 1976.

Schortman, Edward M.; Urban, Patricia A. y Ausec, Marne, "Politics with Style: Identity Formation in Prehispanic Southeastern Mesoamerica", American Anthropologist, 103/2 (2001): 312-330.

Shanks, Michael, Experiencing the past: on the character of archaeology, London, Routledge, 1992.

Shanks, Michael y Tilley, Christopher Y., Re-constructing archaeology: theory and practice, Cambridge, Cambridge University Press, 1987a.

Shanks, Michael y Tilley, Christopher Y., Social theory and archaeology, Cambridge, Polity, $1987 b$. 
Shepard, Anna O., Ceramics for the Archaeologist, Washington, D.C, Carnegie Institution of Washington 1956

Silis García, Omar, El ritual lacustre en los islotes artificiales de la ciénega de Chignahuapan, Santa Cruz Atizapán, Estado de México, México, Escuela Nacional de Antropología e Historia, tesis de licenciatura en Arqueología INEDITA, 2005.

Smith, Anthony, "Towards a global culture", M. Featherstone, Global Culture: Nationalism, Globalization and Modernity, London, Sage, 1990: 171-191.

Smith, Anthony, National identity, London, Penguin, 1991.

Smith, Anthony, "The politics of culture: ethnicity and nationalism", Tim Ingold, Companion Encyclopedia of Anthropology. Humanity, Culture and Social Life, London, Routledge, 1994a: 706-733.

Smith, Anthony, "The problem of national identity: ancient, medieval or modern?", Ethnic and Racial Studies, 17/3 (1994b): 375-399.

Smith, Michael; Novic, Juliana; Kroefges, Peter y Huster, Angela, “A New Map of the Aztec-Period City of Calixtlahuaca in Central Mexico", Antiquity, 81/311 (2007). Project Gallery Online.

Smith, Michael; Novic, Juliana; Kroefges, Peter y Huster, Angela, "Reconocimiento Superficial y Mapeo en Calixtlahuaca”, Expresión Antropológica, 36 (2009): 39-55.

Smith, Michael; Wharton, Jennifer y McCarron, Melissa, "Las ofrendas de Calixtlahuaca”, Expresión Antropológica, 19 (2003): 35-53.

Smyth, Michael P., "Beyond Economic Imperialism: The Teotihuacan Factor in Northern Yucatan", Journal of Anthropological Research, 64/3 (2008): 395-409.

Sökefeld, Martin, "Reconsidering Identity”, Anthropos, 96/2 (2001): 527-544.

Spencer, Martin E., 'Multiculturalism, 'Political Correctness,' and the Politics of Identity", Sociological Forum, 9/4 (1994): 547-567.

Sugiura, Yoko, "En torno a los problemas étnicos en la arqueología regional: la cuenca del alto Lerma en el Posclásico (Parte I: Consideraciones Teóricas)", Anales de Antropología, 28 (1991): 241-270.

Sugiura, Yoko, "Desarrollo histórico del valle de Toluca antes de la conquista española: proceso de conformación pluriétnica", Estudios de Cultura Otopame, 1 (1998a): 99-122.

Sugiura, Yoko, "El valle de Toluca después del ocaso del Estado teotihuacano: el Epiclásico y Postclásico", Ma. Teresa Jarquín y Manuel Miño, Historia General del Estado de México, T1, Geografía y Arqueología, Estado de México, El Colegio Mexiquense, 1998b: 199-259.

Sugiura, Yoko (ed.), La caza, la pesca y la recolección: etnoarqueología del modo de subsistencia lacustre en las ciénegas del Alto Lerma, México, UNAM-IIA, 1998c. 
Sugiura, Yoko, "El valle de Toluca después del ocaso de Teotihuacan", Expresión Antropológica, 11 (2001a): 17-23.

Sugiura, Yoko, "Historia de la Alfarería", Lorenzo Ochoa, Gran Historia de México Ilustrada, México, Editorial Planeta, 2001b: 1-20.

Sugiura, Yoko, “Toluca Region”, Susan Evans y David Webster, Archaeology of Ancient México and Central America: An Encyclopedia, New York/London, Garland Publishing Inc., 2001c: 763-766.

Sugiura, Yoko, "El hombre y la región lacustre en el valle de Toluca: proceso de adaptación en los tiempos prehispánicos", Ernesto Vargas Pacheco, IV Coloquio P. Bosch-Gimpera: El occidente y centro de México, México, IIA/UNAM, 2005a: 303-329.

Sugiura, Yoko, "Reacomodo demográfico y conformación multiétnica en el valle de Toluca durante el Posclásico: una propuesta desde la arqueología”, Linda Manzanilla, Reacomodos demográficos del Clásico al Posclásico en el centro de México, México, UNAM/IIA, 2005b: 175-202.

Sugiura, Yoko, Y atrás quedó las Ciudades de Dioses. Historia de los asentamientos en el valle de Toluca México, UNAM-IIA, 2005c.

Sugiura, Yoko, “¿Continuidad o discontinuidad en la cerámica?: discusión acerca del paso del Clásico al Epiclásico, visto desde el valle de Toluca ", Laura Solar, El fenómeno Coyotlatelco en el centro de México: tiempo, espacio y significado. Memoria del Primer Seminario-Taller sobre Problemáticas Regionales, México, Consejo Nacional para la Cultura y las Artes/INAH/Coordinación Nacional de Arqueología, 2006: 127-162.

Sugiura, Yoko, "Caminando el valle de Toluca: arqueología regional, el legado de William T. Sanders”, Cuicuilco, 16/47 (2009a): 87-111.

Sugiura, Yoko (ed.), La Gente de la Ciénaga en Tiempos Antiguos. La Historia de Santa Cruz Atizapán, México, El Colegio Mexiquense/UNAM-IIA/DGAPA, 2009b.

Sugiura, Yoko, "El valle de Toluca después del ocaso Teotihuacano: el Epiclásico y el Posclásico", Yoko Sugiura, Historia General Ilustrada del Estado de México. Volumen I: Geografía y Arqueología, Toluca, El Colegio Mexiquense, 2011, vol. I: $217-269$.

Sugiura, Yoko, "Reflexiones en torno a los problemas del Epiclásico y el Coyotlatelco", Memoria de la Mesa Redonda: La cerámica del Bajío y regiones aledañas en el Epiclásico: cronología e interacciones, México, CEMCA-INAH-El Colegio de Michoacán AC, en prensa a.

Sugiura, Yoko, "Identidad, cultura material y conocimiento del pasado", Memoria del Simposio Cultura, identidad y patrimonio, Construir el futuro de México, Las ciencias en la UNAM, Mérida, UNAM, en prensa b. 
Sugiura, Yoko y McClung, Emily, "Algunas consideraciones sobre el uso prehispánico de recursos vegetales en la Cuenca del Alto Lerma", Anales de Antropología, 25 (1988): 111-125.

Sugiura, Yoko; Martel, Patricia y Figueroa, Sandra, Atlas etnográfico de la Cuenca alta del río Lerma. Otomíes, mazahuas, matlatzincas y nahuas en los 32 municipios: Vol IV, Toluca, Gobierno del Estado de México, 1997.

Sugiura, Yoko; Barba, Luis; Pérez, Carmen; Ortiz, Agustín y Caballero, Mararita, "Transformaciones del paisaje lacustre: el caso de Santa Cruz Atizapán, Cuenca del Alto Lerma, México Central", Edith Ortiz, Lugar, espacio y paisaje en Arqueología: Mesoamérica y otras áreas culturales: Memoria del VI Coloquio Bosch-Gimpera, México, IIA/UNAM, 2010: 267-298.

Sugiura, Yoko; Zepeda, Elizabeth; Pérez, Carmen y Kabata, Shigueru, "El desarrollo de un asentamiento lacustre en la cuenca alta del río Lerma: el caso de Santa Cruz Atizapán, México Central “, Arqueología Iberoamericana http://www.laiesken.net/ arqueologia/, 5 (marzo-mayo 2010): 5-22. (Verificada: 30/6/2015)

Tilley, Christopher, "Archaeology as socio-political action in the present", V. Pinsky, y A. Wylie, Critical Traditions in Contemporary Archaeology. Essays in the philosophy, history and socio-politics of archaeology, Cambridge, Cambridge University Press, 1989: 104-116.

Tilley, Christopher, Metaphor and Material Culture, Oxford, Blackwell, 1999.

Tomaszewski, Brian M. y Smith, Michael E. "Polities, territory and historical change in Postclassic Matlatzinco (Toluca Valley, central Mexico)", Journal of Historical Geography, 37/1 (2011): 22-39.

Tozzer, A. M., "Excavation of a Site at Santiago Ahuitzotla, D. F., Mexico", Bureau of American Ethnology, Bulletin, 74 (Washington, 1921).

Watson, Patty Jo; Leblanc, Steven A. y Redman, Charles, Explanation In Archaeology: An Explicitly Scientific Approach, New York, Columbia University Press, 1971.

Zepeda, Elizabeth, "Análisis del grupo cerámico Patrón de pulimento en el sitio de Santa Cruz Atizapán”, Estado de México, México, Escuela Nacional de Antropología e Historia/Tesis de Licenciatura, 2009.

Fecha de recepción: 14 de febrero de 2013.

Fecha de aceptación: 26 de mayo de 2013. 


\section{A perspective on the identity-building process in the Pre- Cortesian Toluca Valley in Mexico}

This paper explores identity as a long-term cultural process in which multiple factors intervene at different levels. It argues that both the hegemony and the decline of Teotihuacan influenced the development of collective identities in the Toluca valley, where the study of identity is of particular importance. An analysis of material culture in general, and archeological pottery in particular, enable us to examine the complex web that shaped identity.

Key words: identity; Teotihuacan; Toluca Valley; Epi-Classic; Post-Classic. 